\title{
ON AN APPROACH TO THE EVALUATION OF THE STRENGTH OF A SPATIAL ROD SYSTEM UNDER IMPACT LOADING
}

\author{
I. G. Emel'yanov, V. I. Mironov, A. V. Kuznetsov* \\ Institute of Engineering Science, Ural Branch of the Russian Academy of Sciences, 34, Komsomolskaya st., \\ Ekaterinburg, Russian Federation \\ *Corresponding author. E-mail: Alekseikuz031082@rambler.ru; address for correspondence: ul. Komsomolskaya 34, \\ Ekaterinburg, Russian Federation. Tel.: +7 (343) 375-35-73; fax: 374-53-30
}

An estimation formula for determining the force of interaction between the mass of an electric locomotive and an obstacle is obtained. To determine its parameters, a mathematical model describing the intense strain state of the cabin of an electric locomotive at arrival on an obstacle, including cellular elements, is developed. The loading in the cabin elements is evaluated by the elastic-plastic and dynamic model of material resistance to deformation.

Keywords: thin-walled elements, strength, impact interaction.

\section{References}

1. Normy dlya rascheta $i$ otsenki prochnosti nesushchikh elementov, dinamicheskikh kachestv $i$ vozdeystviy na put ekipazhnoy chasti lokomotivov zheleznykh dorog MPS RF kolei 1520. Vveden 12.01.1998 [Regulations for Calculating and Evaluating the Strength of the Supporting Members and Dynamic Properties of Locomotive Underframes and Their Effects on the Track (Gauge 1520) of the Railways under the Russian Ministry of Railways. Inst. 12.01.1998]. Moscow, FGUP VNIIZhT Publ., 1998. 145 p. (In Russian).

2. Danilenko D.V., Strelchenko A.N. Studying the impact strength of colliding vehicles. TsNIITEI MPS. Zheleznodorozhnyy transport za rubezhom, 2001, series II, iss. 1, pp. 47-49. (In Russian).

3. Demin K.P., Efimov V.P. The strength of colliding vehicles. Zheleznye dorogi mira, 2000, no. 4, pp. 32-36. (In Russian).

4. Kolmogorov V.L., Jonson U., Rid S.R., Korbett G.G. Udarnoe nagruzhenie i razrushenie tverdykh tel [Impact Loading and Fracture of Solids: a Review and a New Theory]. Ekaterinburg, UrO RAN Publ., 2006. 322 p. (In Russian).

5. Fomin V.M., Gulidov A.I., Sapozhnikov G.A. et al. Vusokoskorostnoe vzaimodeistvie tel [High-Speed Interaction of Bodies]. Novosibirsk, SO RAN Publ., 1999. 600 p. (In Russian).

6. Rakhmatulin Kh.A., Demyanov Yu.A. Prochnost pri intensivnykh kratkovremennykh nagruzkakh [Strength under Intensive Momentary Loads]. Moscow, Gos. izd-vo fiz.-mat. lit. Publ., 1961. 393 p. (In Russian).

7. Basov K.A. ANSYS: Spravochnik polzovatelya [ANSYS: User Manual]. Moscow, DMK Press Publ., 2005. 640 p. (In Russian).

8. Emelyanov I.G., Mironov V.I., Kuznetsov A.V. Evaluation of the stress state and lifetime of a shell structure. Problemy mashinostroeniya $i$ nadezhnosti mashin, 2007, no. 5, pp. 57-65. (In Russian).

9. Emelyanov I.G., Kuznetsov A.V., Mironov V.I. A mathematical model describing the stress state of the cabin of a gas turbine locomotive encountering an obstacle. Transport Urala, 2013, no. 4 (39), pp. 71-74. (In Russian). 


\title{
ОБ ОДНОМ ПОДХОДЕ К ОЦЕНКЕ ПРОЧНОСТИ ПРОСТРАНСТВЕННОЙ СТЕРЖ- НЕВОЙ СИСТЕМЫ ПРИ УДАРНОЙ НАГРУЗКЕ
}

\author{
И. Г. Емельянов, В. И. Миронов, А. В. Кузнецов*
}

Федеральное государственное бюджетное учреждение науки Институт машиноведения Уральского отделения Российской академии наук, ул. Комсомольская, 34, Екатеринбург, Российская Федерация

*Ответственный автор. Электронная почта: Alekseikuz031082@rambler.ru; адрес для переписки: ул. Комсомольская 34, Екатеринбург, Российская Федерация; Телефон: +7 (343) 375-35-73; факс: 374-53-30

Получена оценочная формула для определения силы взаимодействия масс электровоза и препятствия. Для определения ее параметров разработана математическая модель, описывающая напряженно-деформированное состояние конструкции кабины электровоза при наезде на препятствие, в том числе и сотовых элементов. Дана оценка значений нагрузок в элементах кабины по упругопластической и динамической модели сопротивления материала деформированию.

Ключевые слова: тонкостенные элементы, прочность, ударное взаимодействие.

\section{1. Введение}

Эксплуатационное разрушение реальных конструкций, состоящих из множества элементов разного типа, сложно описать даже с привлечением самых современных вычислительных средств. Динамический характер нагрузок, возникающих при авариях и катастрофах таких объектов, еще более усложняет задачу. Однако на этапе конструкторской проработки различных технических объектов необходимо знать, как поведет себя объект при различных эксплуатационных нагрузках, в том числе нештатных (например ударных) нагрузках.

Цель данной работы состоит в разработке подхода к оценке напряженного состояния и прочности при ударной нагрузке пространственной балочно-стержневой системы, моделирующей кабину локомотива. На основе современных вычислительных средств разработана математическая модель, позволяющая оценить силу нагружения и напряженнодеформированное состояние конструкции кабины ТЭ8 локомотива $2 Э С 6$ при наезде на препятствие. Рассматривается новый вариант кабины, спроектированный в соответствии с действующими нормами [1] и с учетом опыта конструирования кабин.

Из анализа материалов ОАО «РЖД» и европейских железных дорог $[2,3]$ по аварийным столкновениям поездов известно, что при скорости соударения 20 км/ч сминается лобовая часть кабины локомотива до начала бокового окна, схода тележек с рельсов не наблюдается. При скорости соударения 40 км/ч смятие кабины захватывает и боковое окно, значительно уменьшается внутрикабинное пространство, особенно рабочей зоны локомотивной бригады, и типичным является защемление человека между пультом управления и креслом, травмирование при ударе об элементы конструкции кабины и органы управления, происходит сход локомотива с рельсов.

Ударное нагружение твердых тел и их разрушение - одна из актуальных проблем механики, которая бурно развивается с середины прошлого века $[4,5]$. Для тел небольших размеров и простых форм (удар снарядом или бойком, ковка) существуют аналитические выражения и вычислительные программы (EURDYN-02, ASTARTE, DYNA-2D, DYNA-3D, AUTODIN), позволяющие оценить проникание и разрушение таких тел [4]. Для решения таких задач необходимо наличие информации по физико-механическим параметрам и скорости как ударяемого тела, так и мишени. Многие подходы по решению таких задач базируются на экспериментальных методах исследования. 
Таким образом, при проектировании новой конструкции кабины локомотива необходимо обеспечить безопасность персонала при всех эксплуатационных нагрузках, в том числе при ударном нагружении (наезд на препятствие). При этом безопасность персонала и защита подвижного состава должны быть обеспечены в основном за счет прочности рамы, каркаса кузова, чтобы в случае столкновения деформации имели контролируемый характер, энергия поглощалась за счет пластических деформаций и разрушения буферных зон конструкции.

Например, буферное устройство кабины ТЭ8, защищающее силовой каркас, состоит из принимающей удар лобовой балки с элементами крепления и сотовых энергопоглощающих элементов (рис. 1). Лобовая балка 3, крепящие ее элементы 2 и набор сотовых элементов по замыслу конструкторов кабины должны при столкновении с преградой разрушиться, снижая ударную нагрузку на силовой пояс. При этом передняя балка пояса и боковые ригели 1 не должны получить значительных остаточных деформаций, защищая машиниста, приборную часть, верхнюю и нижнюю обвязку кабины.

В отличие от известных подходов по решению задач на удар, рассматриваемая задача осложняется тем, что информация по механическим параметрам и скорости препятствия (мишени) неизвестна. По требованиям нормативных документов [1] известны только обычные эксплуатационные нагрузки для кабин локомотивов, которые принимаются $3 \mathrm{~g}$ в продольном направлении и 1,25 g в вертикальном направлении (g - ускорение свободного падения). Таким образом, необходимо разработать математическую модель, позволяющую оценить силу нагружения и напряженное состояние конструкции кабины при наезде на препятствие с известными жесткостными и весовыми параметрами препятствия.

В оценочном расчете для решения этой задачи предлагается использовать статический подход, включая в общий алгоритм последовательность краевых задач, разграниченных условиями разрушения отдельных элементов конструкции. После разрушения «слабого звена» фиксируется значение нагрузки и элемент буферного устройства исключается из расчетной схемы для решения новой краевой задачи при возрастающей от нуля нагрузке. При этом нагрузка, при которой появится новое «слабое звено», может оказаться как больше, так и меньше нагрузки, зафиксированной в предыдущем решении. В первом случае следует действовать по алгоритму первого шага, а во втором возможно последовательное разрушение элементов буферного устройства.

В задачах на разрушение особенное значение имеет выбор модели и установление предельного состояния материала. С одной стороны, большие остаточные деформации кабины, наблюдаемые при авариях, предопределяют выбор модели упругопластического упрочняющегося материала. С другой стороны, известно, что разрушение образцов материала при динамических испытаниях носит упругохрупкий характер, а динамический предел текучести может на $30 \%$ превышать статический предел прочности [6]. Поскольку расчет полей напряжений и перемещений реальных конструкций возможен только численными методами, то вторая модель имеет очевидные вычислительные преимущества.

Алгоритм расчета прочности и жесткости конструкции с последовательным исключением «слабого звена» реализован для кабины ТЭ8 с использованием суперкомпьютера «Уран» ИММ УрО РАН. Кабина машиниста локомотива представляет собой сложную пространственную конструкцию, собранную из большого количества гнутых профилей, обтянутых листами обшивки. Материал элементов каркаса и обшивки - сталь 3сп - имеет следующие служебные свойства: модуль упругости $E=2 \cdot 10^{5}$ МПа, предел текучести $\sigma_{T}=240$ МПа, предел прочности $\sigma_{B}=500$ МПа и коэффициент поперечной деформации $v=0,3$.

Ориентировочно можно принять динамический предел текучести $650 \mathrm{MПа,} \mathrm{а} \mathrm{модуль} \mathrm{упроч-}$ нения в упругопластической модели $E_{1}=6 \cdot 10^{3}$ МПа (кривая растяжения для данного материала представлена в виде билинейной кривой с модулями $E$ и $\left.E_{1}\right)$. 
Для определения напряженно-деформированного состояния такой кабины использован вычислительный комплекс ANSYS-14 [7]. Он позволяет исследовать сложные пространственные конструкции в линейной и физически нелинейной постановке $[8,9]$. Поскольку все элементы кабины - тонкостенные конструкции, то в модели использован конечный элемент SHELL-181 и использована опция «большие перемещения».

В математической модели для определения напряженного состояния конструкции кабины в качестве определяющей нагрузки принята статическая горизонтальная единичная сила $Q_{1}=1$ кН, равномерно действующая на переднюю балку кабины. Эта нагрузка имитирует неизвестную нагрузку, которая возникает при столкновении с преградой. Кабина закреплена на четырех вертикальных и двух горизонтальных жестких опорах. Модель была несущественно упрощена для проведения расчетов без изменения основных геометрических размеров и состояла из 378458 конечных элементов. В модели не учитывались перфорация под клепки, болты и панели обшивки кабины. Исходная расчетная схема каркаса кабины представлена на рис. 1.

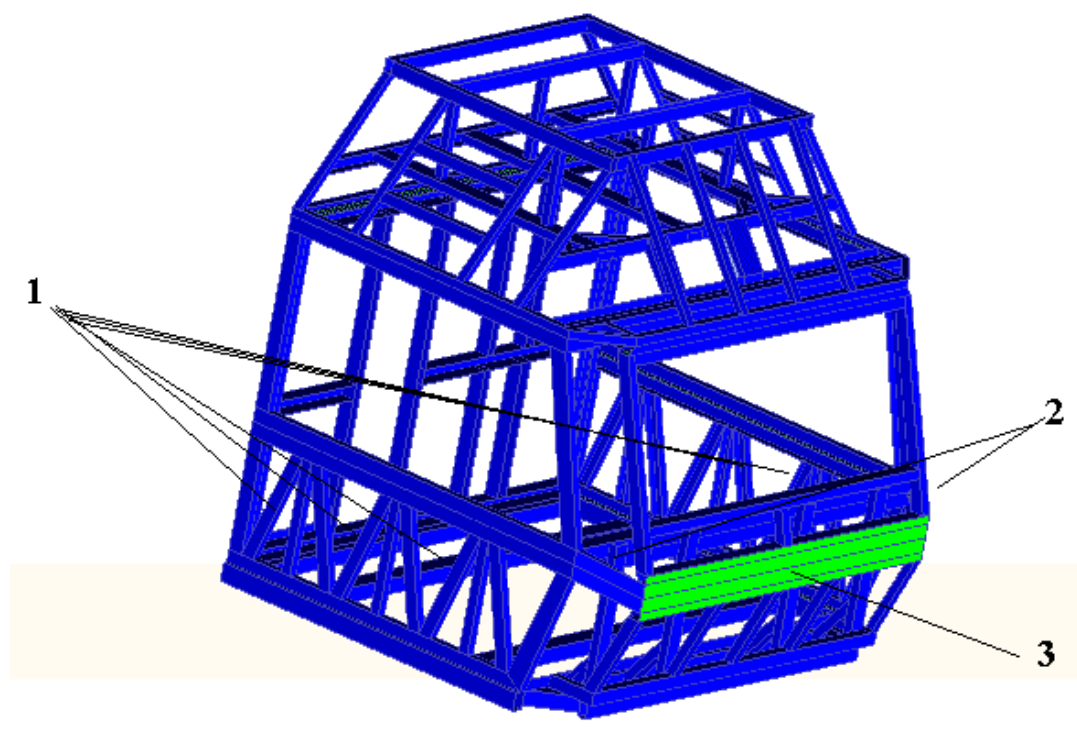

Рис. 1. Чертеж кабины, выполненный в приложении ANSYS

Первым «слабым звеном» оказались наклонные элементы обвязки кабины, в которых опасные напряжения появились при значении суммарной внешней нагрузки 120 кН. Удаление их расчетной схемы мало сказалось на ходе нагрузочной прямой, полученной при решении новой краевой задачи. При нагрузке 300 кН, рекомендованной для оценки прочности кабины, разрушились два центральных блока сотовых элементов. Удаление их расчетной схемы существенно снизило жесткость системы, но опасные напряжения в оставшихся сотовых элементах и лобовой балке возникают при нагрузке вдвое большей. Следовательно, прочность кабины при регламентированной нагрузке обеспечена, но буферное устройство будет частично разрушено. Суммарное перемещение в центре лобовой балки составило при этом 380 мм. Дальнейшее увеличение нагрузки (более 600 кН) приведет к неуправляемому разрушению кабины. На рис. 2 в изометрии показано продольное перемещение кабины $w$ в миллиметрах от регламентированного значения $\mathrm{Q}=30 \mathrm{\kappa H}$.

Оценив предельные нагрузки буферного устройства, необходимо определить силу взаимодействия локомотива с препятствием для установления степени ожидаемых повреждений. Сложность оценки силы взаимодействия кабины с препятствием заключается в неопределенности всех величин, характеризующих препятствие. На железнодорожном переезде вероятнее всего препятствием будет подвижное транспортное средство на пневмоколесном или гусеничном ходу (легковой автомобиль, грузовик, трактор, экскаватор, комбайн). 
opten-aceess jürnal

Масса препятствия может составлять, ориентировочно, от одной до десяти тонн. Скорость движения состава на переезде ограничена интервалом от 20 км/ч до 30 км/ч, или $5,56 \div 8,33 \mathrm{M} / \mathrm{c}$.

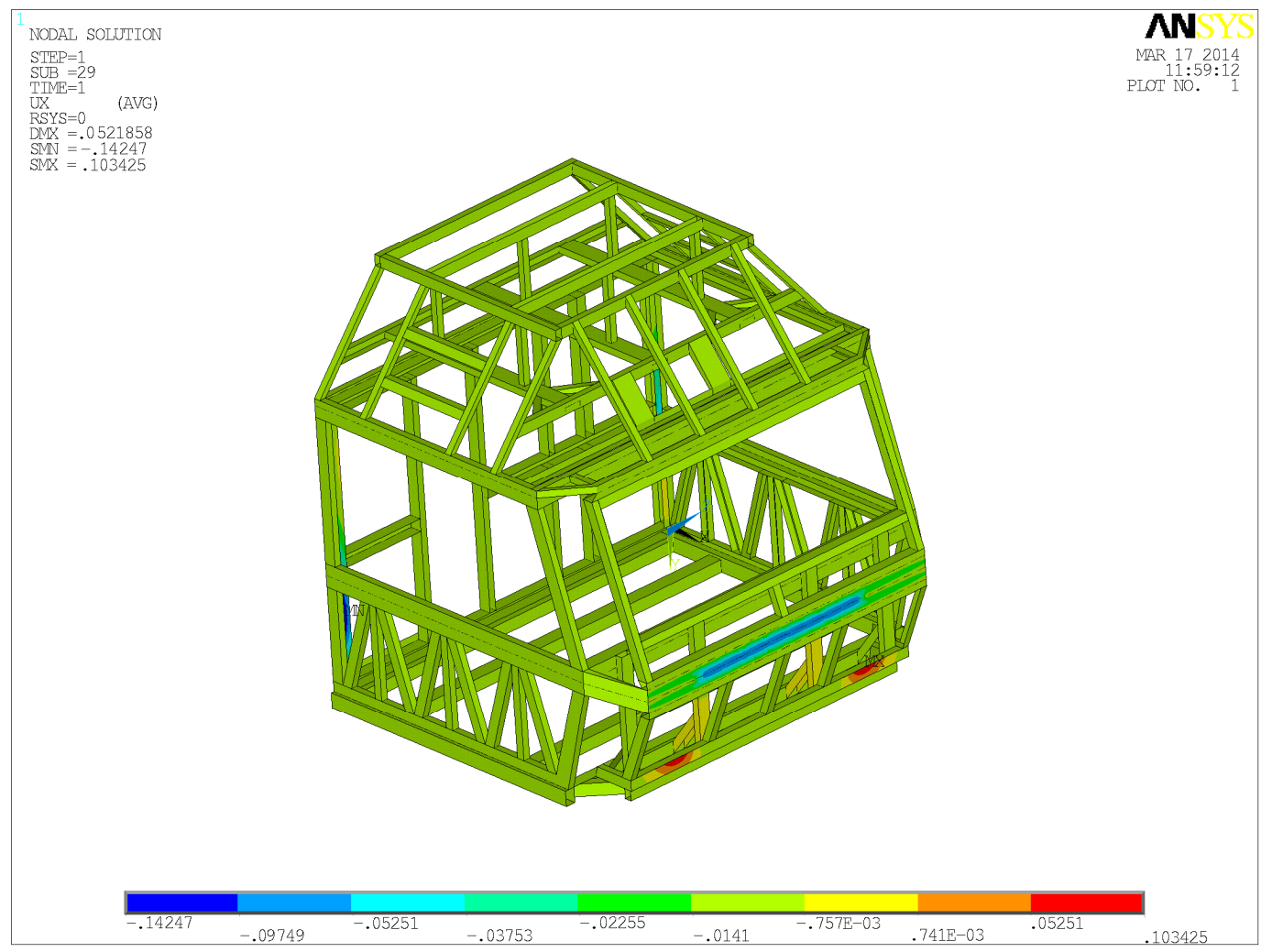

Рис. 2. Продольное перемещение (вдоль движения) кабины в миллиметрах

В динамической задаче по определению силы взаимодействия локомотива с препятствием использована двухмассовая расчетная схема (рис. 3), где $m_{1}$ - масса локомотива, $m_{2}$ - масса препятствия, $\mathrm{P}$ - тяговое усилие, $\mathrm{F}$ - искомая сила упругого взаимодействия, $F_{T}$ - сила трения препятствия с поверхностью переезда. Эквивалентная жесткость пружины при последовательном соединении двух пружин жесткостью $c_{1}$ и $c_{2}$ определяется из выражения

$$
c^{-1}=c_{1}^{-1}+c_{2}^{-1}
$$

где $c_{1}$ - жесткость кабины локомотива с буферным устройством, $c_{2}-$ жесткость препятствия (мишени). Имея решения краевых задач, нетрудно построить нагрузочные прямые для различных расчетных схем и найти по ним жесткость кабины $c_{1}$. Для точного определения жесткости препятствия $c_{2}$ потребуется решение задачи о разрушении препятствия.

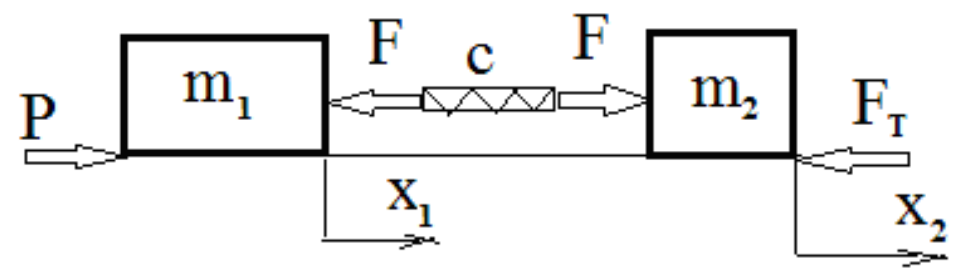

Рис. 3. Расчетная схема динамической задачи 
Задача расчета силы взаимодействия $\mathrm{F}$ решается в два этапа, разделенные условием начала движения второй массы. На первом этапе имеем: $x_{2}=0 ; F \leq F_{T}$; скорость движения газотурбовоза $\dot{x}_{1}=v=$ const; перемещение первой массы $x_{1}=v t$. Первый этап движения заканчивается при выполнении условия равенства сил упругости и трения $F=F_{T}$. Сила упругости в этот момент $F=c x_{1}=c v t_{1 K}$, а сила трения $F_{T}=m_{2} g k$, где $k$-коэффициент сцепления препятствия с полотном переезда. Длительность первого этапа определится выражением $t_{1 K}=m_{2} g k / c v$.

Уравнения движения масс на втором этапе по второму закону Ньютона:

$$
\begin{aligned}
& m_{1} \ddot{x}_{1}=P-F ; \\
& m_{2} \ddot{x}_{2}=F-F_{T} .
\end{aligned}
$$

Дифференцируя дважды по времени выражение для силы упругости $F=c\left(x_{1}-x_{2}\right)$ и подставляя в него выражения ускорений из уравнений (2), выполнив стандартные замены, получим дифференциальное уравнение вынужденных колебаний

$$
\ddot{F}+\omega^{2} F=A,
$$

где квадрат круговой частоты $\omega^{2}=c \frac{m_{1}+m_{2}}{m_{1} m_{2}}$, а правая часть $A=c \frac{P m_{2}+F_{T} m_{1}}{m_{1} m_{2}}$.

Начальные условия для решения неоднородного дифференциального уравнения с постоянными коэффициентами (3): $F_{0}=F_{T} ; \dot{F}_{0}=c\left(\dot{x}_{10}-\dot{x}_{20}\right)=c v$.

Общее решение уравнения (3) имеет вид: $F=C_{1} \operatorname{Cos} \omega t+C_{2} \operatorname{Sin} \omega t+D$, где константа D есть частное решение неоднородного уравнения (3). Подставляя D в исходное уравнение, находим $D=A / \omega^{2}$. Постоянные интегрирования $C_{1}, C_{2}$ находятся из начальных условий.

Решение уравнения (3) дает искомое выражение для силы упругости

$$
F=\frac{\left(F_{T}-P\right) m_{2}}{m_{1}+m_{2}} \operatorname{Cos} \omega t+\frac{c v\left(m_{1} m_{2}\right)^{0,5}}{\left[c\left(m_{1}+m_{2}\right)\right]^{0,5}} \operatorname{Sin} \omega t+\frac{P m_{2}+F_{T} m_{1}}{m_{1}+m_{2}} .
$$

При торможении на переезде масса груженого состава значительно больше, чем масса препятствия ( $\left.m_{1}>>m_{2}\right)$, и выражение (4) упрощается:

$$
F=v \sqrt{c m_{2}} \operatorname{Sin} \omega t+F_{T} .
$$

Максимальная сила упругости при сделанных предположениях оценивается выражением

$$
F_{\max }=v \sqrt{c m_{2}}+F_{T}
$$

Таким образом, для определения максимальной силы взаимодействия необходимо кроме скорости движения состава иметь информацию о весовых и жесткостных параметрах мишени $m_{2}, c_{2}$ и коэффициенте сцепления $k$. В первом оценочном приближении из-за отсутствия информации о мишени положим: $k=0,5 \div 1, m_{2}=(10000 \div 20000) \kappa 2, c_{2}=(0,3 \div 0,5) c_{1}$. Жесткость кабины $\left(c_{1}=2,1 \cdot 10^{6} \mathrm{H} / \mathrm{M}\right)$ найдена по нагрузочной прямой, полученной при 
решении второй краевой задачи. Эквивалентная жесткость по формуле (1) составит $c=(0,25 \div 0,40) \cdot 10^{6} \mathrm{H} / \mathrm{M}$.

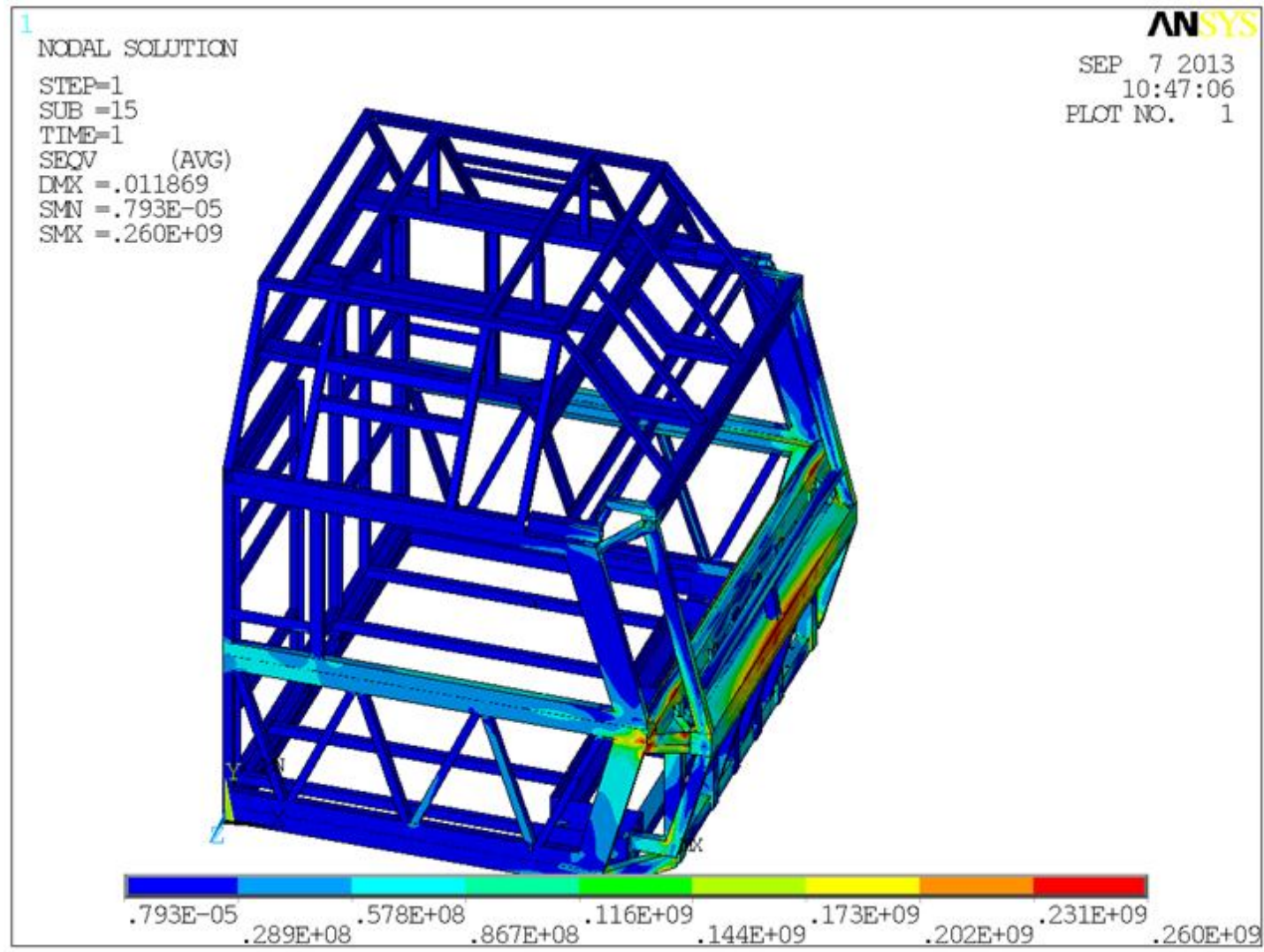

Рис. 4. Напряженное состояние новой кабины при суммарной нагрузке 340 кН (по Мизису)

Максимальная сила взаимодействия локомотива с препятствием по формуле (5) составит $F_{\max }=(300 \div 1050)$ кН. Регламентированная нормами расчета суммарная нагрузка на лобовую балку в 300 кН отвечает нижнему оценочному значению силы взаимодействия. Такая нагрузка неопасна для силового каркаса кабины, который, как отмечалось выше, может выдержать вдвое большую нагрузку (рис. 4). Если в качестве предельного напряжения принять динамический предел текучести, то суммарная нагрузка на лобовую балку в 1000 кН приведет к разрушению буферного устройства, но силовой каркас не разрушится. Чего нельзя утверждать при увеличении скорости движения более 30 км/ч, или при встрече локомотива с еще более тяжелым препятствием более жесткой конструкции.

Работа выполнена при поддержке программы фундаментальных научных исследований Уральского отделения РАН, проект №15-10-1-22.

\section{Литература}

1. Нормы для расчета и оценки прочности несущих элементов, динамических качеств и воздействий на путь экипажной части локомотивов железных дорог МПС РФ колеи 1520. - Введ. 12.01.1998. - М. : ФГУП ВНИИЖТ, 1998. - 145 с.

2. Даниленко Д. В., Стрельченко А. Н. Исследование ударной прочности подвижного состава при столкновениях // ЦНИИТЭИ МПС. Железнодорожный транспорт за рубежом. 2001. - Cер. II, вып. 1. - С. 47-49.

3. Демин К. П., Ефтмов В. П. Прочность подвижного состава при соударении // Железные дороги мира. - 2000. - № 4. - С. 32-36. 
4. Ударное нагружение и разрушение твердых тел / В. Л. Колмогоров, У. Джонсон, С. Р. Рид, Г. Г. Корбетт. - Екатеринбург : УрО РАН, 2006. - 322 с.

5. Высокоскоростное взаимодействие тел / В. М. Фомин, А. И. Гулидов, Г. А. Сапожников и др. - Новосибирск : СО РАН, 1999. - 600 с.

6. Рахматулин Х. А., Демьянов Ю. А. Прочность при интенсивных кратковременных нагрузках. - М. : Гос. изд-во физ.-мат. лит., 1961. - 393 с.

7. Басов К. A. ANSYS: Справочник пользователя. - М. : ДМК Пресс, 2005. - 640 с.

8. Емельянов И. Г., Миронов В. И., Кузнецов А. В. Определение напряженного состояния и ресурса оболочечной конструкции // Проблемы машиностроения и надежности машин. - 2007. - № 5. - С. 57-65.

9. Емельянов И. Г., Кузнецов А. В., Миронов В. И. Математическая модель, описывающая напряженное состояние кабины газотурбовоза при встрече с препятствием // Транспорт Урала. - 2013. - № 4 (39). - С. 71-74. 